$\mathrm{SINP} / \mathrm{TNP} / 99-25$

hep-ph/9907432

\title{
Neutrino mass and magnetic moment in supersymmetry without $R$-parity in the light of recent data
}

\author{
Gautam Bhattacharyya ${ }^{1,2}$, H.V. Klapdor-Kleingrothaus ${ }^{1}$, Heinrich Päs ${ }^{1}$ \\ 1 Max-Planck-Institut für Kernphysik, P.O. Box 103980, D-69029 Heidelberg, Germany \\ 2 Saha Institute of Nuclear Physics, 1/AF Bidhan Nagar, Calcutta 700064, India
}

\begin{abstract}
We consider the generation of neutrino Majorana mass and transition magnetic moment by the lepton-number violating $\lambda$ and/or $\lambda^{\prime}$ couplings in $R$-parity-violating supersymmetric models. We update (and improve) the existing upper limits on the relevant couplings using the most recent data on neutrino masses and mixings, indicating also the possible improvement by the GENIUS project. We study the implication of this update on the induced neutrino magnetic moment.
\end{abstract}

Supersymmetry without $R$-parity [1] provides an elegant mechanism for generating neutrino (Majorana) masses and mixings. In these models, there are mainly two sources of neutrino mass generation. In one scenario, products of trilinear $\lambda$ and/or $\lambda^{\prime}$ couplings generate a complete neutrino mass matrix through one-loop self-energy graphs [2, 3]. In this framework, the diagrams that generate the masses also generate magnetic moments by insertion of photons to the internal lines. In the other scenario, the bilinear $R$ parity-violating (RPV) terms induce sneutrino vacuum expectation values (VEV's) allowing neutrinos to mix with the neutralinos. In this mechanism only one physical neutrino becomes massive [4. In the present analysis, although we concentrate mainly on the trilinear $L$-violating couplings, we also comment on the possible impact of the bilinear parameters. We use the recent data on atmospheric [5] and solar [6] neutrinos, the measurement of $\nu_{e}$ mass in the Troitsk [7] and Mainz 8] tritium beta-decay experiments, and the measurement of the effective neutrino mass $\left\langle m_{\nu}\right\rangle$ in the Heidelberg - Moscow ${ }^{76}$ Ge experiment [9], to update (and improve) the existing limits 110 on many different combinations of the trilinear couplings from their contribution to neutrino masses. We also calculate the magnetic moment of the neutrino, which has an intimate connection to its mass (for previous studies of neutrino magnetic moments in RPV models, see $[11,12])$.

Generally two types of magnetic moment may arise: (i) the Dirac-type magnetic moment that rotates $\nu_{e L}$ to $\nu_{e R}$, and (ii) the transition magnetic moment that takes $\nu_{e L}$ to $\nu_{\mu R}^{c}$. If one adds a right-handed singlet neutrino to the standard model (SM), a non-zero Dirac mass of the neutrino implies a non-zero Dirac-type magnetic moment, given by 13 .

$$
\mu_{\nu}=\frac{3 e G_{F} m_{\nu}}{8 \pi^{2} \sqrt{2}}=3 \times 10^{-19}\left(\frac{m_{\nu}}{1 \mathrm{eV}}\right) \mu_{B},
$$

where $\mu_{B}=e / 2 m_{e}$ is the Bohr magneton. The supersymmetric contribution to the Dirac-type magnetic moment could at most be comparable to the above value [14]. On the other hand, a Majorana neutrino, massive or massless, cannot possess a diagonal magnetic moment due to the CPT theorem. It may only have a transition magnetic moment between two species having opposite $C P$ eigenvalues. In supersymmetric models without a right-handed neutrino, the RPV couplings can generate only $\Delta L=2$ Majorana masses. The magnetic moment they induce is therefore only transitional.

The $L$-violating part of the RPV superpotential can be written as

$$
\mathcal{W}_{R P V}=\frac{1}{2} \lambda_{i j k} L_{i} L_{j} E_{k}^{c}+\lambda_{i j k}^{\prime} L_{i} Q_{j} D_{k}^{c}+\mu_{i} L_{i} H_{u},
$$

where $i, j$ and $k$ are quark and lepton generation indices. In Eq. (2), $L_{i}$ and $Q_{i}$ are $\mathrm{SU}(2)$-doublet lepton and quark superfields, $E_{i}^{c}$ and $D_{i}^{c}$ are $\mathrm{SU}(2)$-singlet charged lepton and down-quark superfields, and $H_{u}$ is the Higgs superfield responsible for the mass generation of the up-type quarks, respectively. There are 9 
$\lambda$-type (due to an antisymmetry in the first two generation indices), $27 \lambda^{\prime}$-type and $3 \mu_{i}$ couplings. Stringent upper limits exist on all these couplings from different experiments 10, 15.

We first consider the effects of the $\lambda^{\prime}$ interactions. The relevant part of the Lagrangian can be written as

$$
-\mathcal{L}_{\lambda^{\prime}}=\lambda_{i j k}^{\prime}\left[\bar{d}_{k} P_{L} \nu_{i} \tilde{d}_{j L}+\bar{\nu}_{i}^{c} P_{L} d_{j} \tilde{d}_{k R}^{*}\right]+\text { h.c. }
$$

Majorana mass terms for the left-handed neutrinos, given by $\mathcal{L}_{M}=-\frac{1}{2} m_{\nu_{i i^{\prime}}} \bar{\nu}_{L i} \nu_{R i^{\prime}}^{c}+$ h.c., are generated at one loop. Figs. 1(a) and 1(b) show the corresponding diagrams. The induced masses are given by

$$
m_{\nu_{i i^{\prime}}} \simeq \frac{N_{c} \lambda_{i j k}^{\prime} \lambda_{i^{\prime} k j}^{\prime}}{16 \pi^{2}} m_{d_{j}} m_{d_{k}}\left[\frac{f\left(m_{d_{j}}^{2} / m_{\tilde{d}_{k}}^{2}\right)}{m_{\tilde{d}_{k}}}+\frac{f\left(m_{d_{k}}^{2} / m_{\tilde{d}_{j}}^{2}\right)}{m_{\tilde{d}_{j}}}\right],
$$

where $f(x)=(x \ln x-x+1) /(x-1)^{2}$. Here, $m_{d_{i}}$ is the down quark mass of the $i$ th generation inside the loop, $m_{\tilde{d}_{i}}$ is an average of $\tilde{d}_{L i}$ and $\tilde{d}_{R i}$ squark masses, and $N_{c}=3$ is the colour factor. In deriving Eq. (位), we assumed that the left-right squark mixing terms in the soft part of the Lagrangian are diagonal in their physical basis and proportional to the corresponding quark masses, i.e. $\Delta m_{\mathrm{LR}}^{2}(i)=m_{d_{i}} m_{\tilde{d}_{i}}$. At this point, a remark about the quark mixing angles is in order. These angles are supposed to appear in the loop amplitudes under consideration. The reason behind this is that the RPV couplings have been written in the flavour basis, while the states that propagate inside the loop are in their mass basis. However, we have ignored this small difference in order not to complicate the discussion unnecessarily. On the other hand, the neutrinos may have large mixings and so the difference between their flavour and mass eigenstates cannot be ignored.

Now one can study the electromagnetic properties of the neutrino, namely the magnetic moment, by attaching photons to the internal lines of the loops in Figs. 1(a) and 1(b). One can express the effective Hamiltonian as

$$
\mathcal{H}_{\mathrm{eff}}=\mu_{\nu_{i i^{\prime}}} \bar{\nu}_{L i}\left(\frac{\sigma^{\alpha \beta}}{2}\right) \nu_{R i^{\prime}}^{c} F_{\beta \alpha}
$$

where $\mu_{\nu_{i i^{\prime}}}$ is the transition magnetic moment between the states $\nu_{i}$ and $\nu_{i^{\prime}}$. It is given by

$$
\mu_{\nu_{i i^{\prime}}} \simeq\left(1-\delta_{i i^{\prime}}\right) \frac{N_{c} \lambda_{i j k}^{\prime} \lambda_{i^{\prime} k j}^{\prime}}{4 \pi^{2}} m_{e} Q_{d} m_{d_{j}} m_{d_{k}}\left[\frac{g\left(m_{d_{j}}^{2} / m_{\tilde{d}_{k}}^{2}\right)}{m_{\tilde{d}_{k}}^{3}}-\frac{g\left(m_{d_{k}}^{2} / m_{\tilde{d}_{j}}^{2}\right)}{m_{\tilde{d}_{j}}^{3}}\right] \mu_{B}
$$

where $g(x)=(1+x) \ln x /(x-1)^{3}-2 /(x-1)^{2}$, and the $\left(1-\delta_{i i^{\prime}}\right)$ factor indicates that for $i=i^{\prime}$ the magnetic moment is zero. It is interesting to note that for $j=k$, even if there is a contribution to the neutrino mass, the transitional magnetic moment vanishes.

Since all down-type quarks are much lighter than the squarks, $x \ll 1$ (for all generations) is a good approximation. This implies $f(x) \simeq 1$, and $g(x) \simeq-\ln x-2$. We now consider the following two cases. In the first case, the down squarks of $j$ th and $k$ th generation are degenerate, i.e. $m_{\tilde{d}_{j}}=m_{\tilde{d}_{k}} \equiv \tilde{m}$. Then

$$
\mu_{\nu_{i i^{\prime}}} \simeq\left(1-\delta_{i i^{\prime}}\right) 8 m_{\nu_{i i^{\prime}}} \frac{m_{e} Q_{d}}{\tilde{m}^{2}} \ln \left(\frac{m_{d_{j}}}{m_{d_{k}}}\right) \mu_{B}
$$

In the second case, the down squark of the $j$ th (say) generation is much heavier than that of the $k$ th generation; so only one term within the square bracket of Eq. (6) effectively contributes. Then

$$
\mu_{\nu_{i i^{\prime}}} \simeq\left(1-\delta_{i i^{\prime}}\right) 8 m_{\nu_{i i^{\prime}}} \frac{m_{e} Q_{d}}{m_{\tilde{d}_{k}}^{2}}\left[\ln \left(\frac{m_{\tilde{d}_{k}}}{m_{d_{j}}}\right)-1\right] \mu_{B}
$$

With $\lambda$-type interactions, one obtains exactly similar results as in Eqs. (3) to (8). The quarks and squarks in these equations will be replaced by the leptons and sleptons of the corresponding generations. The colour factor $N_{c}=3$ in Eqs. (4) and (6) and $Q_{d}$ in Eqs. (6) to (8) would be replaced by 1 and $Q_{e}$ respectively. We do not explicitly write them down. We draw the attention of the readers to the fact that Barbieri et al. 12 have overlooked a part of the Lagrangian associated with a given pair of $\lambda$ while presenting their analytic formulae and the corresponding estimates. Since they assumed that sleptons of 
different generations are fairly degenerate, (a) their expression of the neutrino mass [Eq. (3a) of [12] ] should be multiplied by a factor of 2, and (b) their expressions of the magnetic moment [Eqs. (3b) and (4) of [12], which are similar to our Eq. (8)] should correspond to our Eq. (7). Indeed these details do not change the order-of-magnitude estimates they have presented.

In Table 1, we have presented the upper limits on different product couplings calculated from the most recent bounds on the different entries of the neutrino mass matrix (in flavour space). We have also presented the transition magnetic moments between different flavours derived using the most stringent constraints on the relevant product couplings. Among the different entries of the flavour space mass matrix, only the ee-term has a direct experimental bound $m_{e e}<0.2 \mathrm{eV}$ obtained by the Heidelberg - Moscow neutrinoless double-beta decay experiment [9. The planned GENIUS project in the 1 and 10 ton versions [16] would be sensitive to $m_{e e}$ as low as 0.01 and $0.001 \mathrm{eV}$ respectively. We have displayed their possible impact in Table 1. To constrain the other entries of the mass matrix we use (a) the limit on the lightest neutrino mass eigenstate $m_{1}<2.5 \mathrm{eV}$ obtained by the Troitsk tritium beta-decay experiment [7], in conjunction with (b) the oscillation solutions of the atmospheric and solar neutrino problems [5, 6], implying

$$
\Delta m_{12}=\Delta m_{\odot} \ll \Delta m_{13}=\Delta m_{a t m} \ll \mathcal{O}(1 \mathrm{eV})<m_{1},
$$

in a three neutrino framework. Hence from unitarity an upper bound $m_{\nu_{i i^{\prime}}}<2.5 \mathrm{eV}$ can be obtained for any flavour combination $\left(i, i^{\prime}\right)$. A larger spectrometer planned for tritium beta-decay experiment [17] would bring the limit down to $m_{\nu_{i i^{\prime}}}<1 \mathrm{eV}$. While most of the existing bounds are simply the products of the bounds on the individual couplings given in [18], there are some combinations which receive stringent limits from $\mu$ conversion in nuclei [19]. The chirality flips in our Figs. 1(a) and 1(b) explain why with heavier fermions inside the loop the bounds are tighter. For this reason, we have presented the bounds only for $j, k=2,3$. In the process, constraints on the individual couplings $\lambda_{122}, \lambda_{133}, \lambda_{233}, \lambda_{322}, \lambda_{i 33}^{\prime}$ and $\lambda_{i 22}^{\prime}$ (for all $i$ ) have improved quite significantly. The most stringent upper limits are then employed to derive the maximum magnetic moment that RPV models can induce, using Eq. (7) for the degenerate case and Eq. (8) for the hierarchical case. Since Eq. (8) is asymmetric in $j$ and $k$, both cases $m_{\tilde{d}_{j}} \gg m_{\tilde{d}_{k}}$ and $m_{\tilde{d}_{k}} \gg m_{\tilde{d}_{j}}$ are separately examined. For numerical purpose, we have assumed the mass of whatever scalar is relevant to be $100 \mathrm{GeV}$ throughout, to be consistent with common practice and, in particular, to compare with the old bounds. While for sleptons this sounds a reasonable approximation, for squarks the present lower limit, even in RPV scenario, is around $250 \mathrm{GeV}$ [20]. In any case, for different squark masses one can easily derive the appropriate bounds by straightforward scaling. It should be noted that the product couplings under consideration contribute to charged lepton masses as well. But with the present limits those contributions are too small to be of any relevance.

The bilinear couplings (the last term of Eq. (2D) could be another source of neutrino mass generation. Only one of the neutrino physical states becomes massive if $\mu_{\alpha}$ and $\left\langle L_{\alpha}\right\rangle$ (where, $\alpha=0, i$ ) are not aligned [4. Here, $\mu_{0} \equiv \mu$ (note, $\mu H_{d} H_{u}$ appears in the $R$-parity-conserving superpotential) and $L_{0} \equiv H_{d}$ (the Higgs superfield responsible for down quark mass generation). The misalignment induces sneutrino VEV allowing neutrinos to mix with the neutralinos, and, as a result, a neutrino mass is generated. The implications of the bilinear couplings in the light of Super-Kamiokande (SK) data and neutrinoless double-beta decay have been studied in Ref. [15] (in a restricted scenario) and Ref. [21], respectively. Since the neutrino mass is induced at tree level, this interaction cannot generate any neutrino magnetic moment. In this context, an interesting idea in the framework of supergravity models has been proposed in Ref. 22. The presence of trilinear RPV interaction at a high scale induces bilinear RPV terms in the effective potential at the weak scale. The sneutrino VEV induced this way causes neutrino-neutralino mixing. The neutrino mass generated through this mixing dominates over the loop mass (due to the presence of a large logarithm). The constraints on $\lambda_{i k k}^{\prime}$ obtained in this specific scheme are somewhat stronger (barring accidental cancellation) than obtained from direct loop induced processes.

At this point, a few comments on the existing bounds on neutrino magnetic moment are in order. From SK solar neutrino data, a limit $\mu_{\nu_{e}}<1.6 \times 10^{-10} \mu_{B}$ has recently been obtained [23]. From SN1987A a bound $\mu_{\nu_{e}}<10^{-10} \mu_{B}$ was obtained [24], although the bound from supernova applies only to Dirac-type magnetic moments. The present experimental upper limits on $\mu_{\nu_{e}}<1.8 \times 10^{-10} \mu_{B}$ and $\mu_{\nu_{\mu}}<7.4 \times 10^{-10} \mu_{B}$ have been obtained from neutral current scattering data [25, 26]. For the tau neutrino, a new bound $\mu_{\nu_{\tau}}<1.3 \times 10^{-7} \mu_{B}$ has recently been obtained from SK data [27]. In the near future, a new detector 
of the MUNU Collaboration [28] installed near one of the reactors of the Bugey power station should be sensitive to transition magnetic moments (relating $\nu_{e}$ to other states) of order $(2-3) \times 10^{-11} \mu_{B}$.

Since a large neutrino magnetic moment $\left(10^{-10}-10^{-11} \mu_{B}\right)$ can provide an elegant solution to the solar neutrino problem [29], by flipping $\nu_{e}$ to undetectable species, it has always been a theoretically challenging exercise [30] to build models which can generate a sizable magnetic moment of the neutrino, keeping its mass small. But unless some additional symmetry is invoked, it is difficult to endow a neutrino with a large magnetic moment, since the same diagrams that induce neutrino masses also generate magnetic moments (by insertion of photons to internal lines). The task therefore is to look for some continuous or discrete horizontal symmetry that would allow the magnetic moment but suppress the mass of the neutrino. As an example, Voloshin [31] considered an $\mathrm{SU}(2)$ horizontal symmetry, acting on the $\left(\nu, \nu^{c}\right)$ doublet, under which the neutrino Majorana mass term transforms as a triplet and is therefore prohibited in the limit of exact symmetry; the neutrino magnetic moment, on the other hand, transforms as a singlet and hence can be large. In the context of RPV models, imposing a horizontal symmetry was shown to provide at most an order of magnitude enhancement [12]. Since the limits on RPV couplings induced magnetic moment we obtain in the present analysis, are at least a few orders of magnitude below the present level of experimental sensitivity and the requirement for the solar neutrino solution, it is unlikely that any additional symmetry imposed on the RPV Lagrangian could provide the necessary enhancement to close the gap.

To conclude, we have improved the upper limits on many individual and product couplings of the $\lambda$ and $\lambda^{\prime}$-types, from their contribution to neutrino masses, using the most recent data. We have also indicated how these limits will be further strengthened once the planned experiments are realized. Attaching photons to the internal lines of the loop diagrams that generate neutrino masses also induces transition magnetic moments. The maximum induced magnetic moments we obtain are too small to be tested in the present laboratory experiments.

G.B. acknowledges warm hospitality at the Max-Planck-Institut für Kernphysik, Heidelberg.

\section{References}

[1] G. Farrar, P. Fayet, Phys. Lett. 76B, 575 (1978); S. Weinberg, Phys. Rev. D 26, 287 (1982); N. Sakai, T. Yanagida, Nucl. Phys. B 197, 533 (1982); C. Aulakh, R. Mohapatra, Phys. Lett. 119B, 136 (1982).

[2] S. Dimopoulos, L. Hall, Phys. Lett. B207, 210 (1987); R. Godbole, P. Roy, X. Tata, Nucl. Phys. B 401, 67 (1993).

[3] For recent studies, see M. Drees, S. Pakvasa, X. Tata and T. ter Veldhuis, Phys. Rev. D 57, 5335 (1998); S. Rakshit, G. Bhattacharyya, A. Raychaudhuri, Phys. Rev. D 59, 091701 (1999); R. Adhikari, G. Omanovic, Phys. Rev. D 59, 073003 (1999); O. Kong, Mod. Phys. Lett. A 14, 903 (1999); E.J. Chun, S.K. Kang, C.W. Kim, U.W. Lee, Nucl. Phys. B 544, 89 (1999); K. Choi, K. Hwang, E.J. Chun, hep-ph/9811363; A. Joshipura, S. Vempati, hep-ph/9903435.

[4] A.Y. Smirnov, F. Vissani, Nucl. Phys. B 460, 37 (1996); E. Nardi, Phys. Rev. D 55, 5772 (1997); T. Banks, Y. Grossman, E. Nardi, Y. Nir, Phys. Rev. D 52, 5319 (1995); R. Hempfling, Nucl. Phys. B 478 , 3 (1996); H. Nilles, N. Polonsky, Nucl. Phys. B 484, 33 (1997); C. Liu, Mod. Phys. Lett. A 12, 329 (1997); B. Mukhopadhyaya, S. Roy, F. Vissani, Phys. Lett. B 443, 191 (1998); D.E. Kaplan, A. Nelson, hep-ph/9901254; J. Valle, hep-ph/9712277.

[5] Super-Kamiokande Collaboration, Y. Fukuda et al., Phys. Rev. Lett. 81, 1562 (1998).

[6] J.N. Bahcall, M.H. Pinsonneault, Rev. Mod. Phys. 67, 781 (1995); J.N. Bahcall, S. Basu, M.H. Pinsonneault, Phys. Lett. B 433, 1 (1998).

[7] V.M. Lobashov (for Troitsk Collaboration), Proc. of Neutrino '98, Takayama, Japan, June 1998.

[8] C. Weinheimer (Mainz Collaboration), private communication.

[9] L. Baudis et al., Phys. Rev. Lett. 83, 41 (1999); H.V. Klapdor-Kleingrothaus, hep-ex/9901021, Proc. Int. Conf. on Lepton- and Baryon Number Non-Conservation, Trento, Italy, April 1998, IOP, Bristol, 1999. 
[10] For recent reviews, see G. Bhattacharyya, hep-ph/9709395, Proc. BEYOND 97, Castle Ringberg, Germany, June 1997, eds. H.V. Klapdor-Kleingrothaus, H. Päs, IOP, Bristol, 1998 (and its update to appear in the Proc. of the Int. WEIN98 Conf., Santa Fe, USA, June 1998); Nucl. Phys. Proc. Suppl. 52A, 83 (1997); H. Dreiner, hep-ph/9707435; R. Barbier et al., hep-ph/9810232; P. Roy, hep$\mathrm{ph} / 9712520$.

[11] K.S. Babu, R.N. Mohapatra, Phys. Rev. Lett. 64, 1705 (1990); K. Enqvist, A. Masiero, A. Riotto, Nucl. Phys. B 373, 95 (1992); E. Roulet, D. Tommasini, Phys. Lett. B 256, 218 (1991).

[12] R. Barbieri, M. Guzzo, A. Masiero, D. Tommasini, Phys. Lett. B 252, 251 (1990).

[13] K. Fujikawa, R. Shrock, Phys. Rev. Lett. 45, 963 (1980).

[14] K.L. Ng, Z. Phys. C 48, 289 (1990).

[15] V. Bednyakov, A. Faessler, S. Kovalenko, Phys. Lett. B 442, 203 (1998)

[16] H.V. Klapdor-Kleingrothaus, Proc. BEYOND 97 [10]; J. Hellmig, H.V. Klapdor-Kleingrothaus, Z. Phys. A 359, 351 (1997); H.V. Klapdor-Kleingrothaus, M. Hirsch, Z. Phys. A 359, 361 (1997); H.V. Klapdor-Kleingrothaus, J. Hellmig, M. Hirsch, J. Phys. G 24, 483 (1998); H.V. Klapdor-Kleingrothaus, Int. J. Mod. Phys. A 23, 3953 (1998).

[17] J. Bonn et al., Nucl. Instr. Meth. A 421, 256 (1999).

[18] Bhattacharyya in [10] (to appear in the Proc. of the International WEIN98 Conference).

[19] A. Faessler, T.S. Kosmas, S. Kovalenko, J.D. Vergados, hep-ph/9904335; K. Huitu, J. Maalampi, M. Raidal, A. Santamaria, Phys. Lett. B 430, 355 (1998).

[20] B. Abott et al., D0 Collaboration, hep-ex/9907019.

[21] M. Hirsch, J. Valle, hep-ph/9812463; A. Faessler, S. Kovalenko, F. Simkovic, Phys. Rev. D 58, 055004 (1998).

[22] Joshipura, Vempati in [3].

[23] J.F. Beacom, P. Vogel, hep-ph/9907383.

[24] J.M. Lattimer, J. Cooperstein, Phys. Rev. Lett. 61, 23 (1988); R. Barbieri, R.N. Mohapatra, Phys. Rev. Lett. 61, 27 (1988).

[25] The Review of Particle Physics, C. Caso et al., Eur. Phys. J. C 3, 1 (1998) (and 1999 off-year partial web-update).

[26] J.E. Kim, Phys. Rev. Lett. 41, 360 (1978); hep-ph/9904312.

[27] S.N. Gninenko, Phys. Lett. B 452, 414 (1999); M. Maltoni, M.I. Vysotsky, hep-ph/9804464.

[28] Y. Declais, private communication.

[29] A. Cisneros, Astrophys. Space Sci. 10, 87 (1971); L.B. Okun, M.B. Voloshin, M.I. Vysotsky, Sov. Phys. JETP 64, 446 (1986). For a pedagogic discussion, see R.N. Mohapatra, P.B. Pal, "Massive neutrinos in Physics and Astrophysics" (World Scientific, Singapore, Second Edition, 1998).

[30] M. Voloshin, Sov. J. Nucl. Phys. 48, 512 (1988); R. Barbieri, R.N. Mohapatra, Phys. Lett. B 218, 225 (1989); K.S. Babu, R.N. Mohapatra, Phys. Rev. Lett. 63, 228 (1989); Phys. Rev. D 43, 2278 (1991); G. Ecker, W. Grimus, H. Neufeld, Phys. Lett. B 232, 217 (1989); D. Chang, W. Keung, G. Senjanovic, Phys. Rev. D 42, 1599 (1990); M. Leurer, N. Marcus, Phys. Lett. B 237, 81 (1990); J.C. Montero, V. Pleitez, hep-ph/9907212.

[31] Voloshin in [30]. 
Table 1: Correlation among neutrino mass bounds, upper limits on RPV couplings and neutrino magnetic moments. We have used $m_{d}=9 \mathrm{MeV}, m_{s}=170 \mathrm{MeV}, m_{b}=4.4 \mathrm{GeV}$ 25]. The potential of the planned GENIUS project in the 1 and 10 ton versions is also exhibited. If not mentioned, the previous bounds are products of bounds on individual couplings given in [18]. For $\lambda$-products, $m_{\tilde{d}}$ should be read as $m_{\tilde{e}}$. The relevant scalars are always assumed to have a common mass of $100 \mathrm{GeV}$.

\begin{tabular}{|c|c|c|c|c|c|}
\hline$\lambda_{i j k}^{\left({ }^{\prime}\right)} \lambda_{i^{\prime} k j}^{\left({ }^{\prime}\right)}$ & $\begin{array}{c}\text { Our } \\
\text { Bounds }\end{array}$ & $\begin{array}{l}\text { Previous } \\
\text { Bounds }\end{array}$ & $\begin{array}{c}\mu_{\nu_{i i}{ }^{\prime}} \\
\left(m_{\tilde{d}_{j}}=m_{\tilde{d}_{k}}\right) \\
\end{array}$ & $\begin{array}{c}\mu_{\nu_{i i}{ }^{\prime}} \\
\left(m_{\tilde{d}_{j}} \gg m_{\tilde{d}_{k}}\right) \\
\end{array}$ & $\begin{array}{c}\mu_{\nu_{i i i^{\prime}}} \\
\left(m_{\tilde{d}_{k}} \gg m_{\tilde{d}_{j}}\right) \\
\end{array}$ \\
\hline \multicolumn{6}{|l|}{$m_{e e}<0.2 \mathrm{eV}$} \\
\hline$\lambda_{133}^{\prime} \lambda_{133}^{\prime}$ & $3.0 \cdot 10^{-8}$ & $4.9 \cdot 10^{-7}$ & 0 & 0 & 0 \\
\hline$\lambda_{132}^{\prime} \lambda_{123}^{\prime}$ & $7.5 \cdot 10^{-7}$ & $1.6 \cdot 10^{-2}$ & 0 & 0 & 0 \\
\hline$\lambda_{122}^{\prime} \lambda_{122}^{\prime}$ & $1.8 \cdot 10^{-5}$ & $4.0 \cdot 10^{-4}$ & 0 & 0 & 0 \\
\hline$\lambda_{133} \lambda_{133}$ & $5.3 \cdot 10^{-7}$ & $9.0 \cdot 10^{-6}$ & 0 & 0 & 0 \\
\hline$\lambda_{132} \lambda_{123}$ & $8.7 \cdot 10^{-6}$ & $2.0 \cdot 10^{-3}$ & 0 & 0 & 0 \\
\hline$\lambda_{122} \lambda_{122}$ & $1.4 \cdot 10^{-4}$ & $1.6 \cdot 10^{-3}$ & 0 & 0 & 0 \\
\hline$m_{e e}<0.01(0.001) \mathrm{eV}$ & [GENIUS 1(10)t] & & & & \\
\hline$\lambda_{133}^{\prime} \lambda_{133}^{\prime}$ & $1.5 \cdot 10^{-9(-10)}$ & $4.9 \cdot 10^{-7}$ & 0 & 0 & 0 \\
\hline$\lambda_{132}^{\prime} \lambda_{123}^{\prime}$ & $3.7 \cdot 10^{-8(-9)}$ & $1.6 \cdot 10^{-2}$ & 0 & 0 & 0 \\
\hline$\lambda_{122}^{\prime} \lambda_{122}^{\prime}$ & $9.2 \cdot 10^{-7(-8)}$ & $4.0 \cdot 10^{-4}$ & 0 & 0 & 0 \\
\hline$\lambda_{133} \lambda_{133}$ & $2.6 \cdot 10^{-8(-9)}$ & $9.0 \cdot 10^{-6}$ & 0 & 0 & 0 \\
\hline$\lambda_{132} \lambda_{123}$ & $4.3 \cdot 10^{-7(-8)}$ & $2.0 \cdot 10^{-3}$ & 0 & 0 & 0 \\
\hline$\lambda_{122} \lambda_{122}$ & $7.1 \cdot 10^{-6(-7)}$ & $1.6 \cdot 10^{-3}$ & 0 & 0 & 0 \\
\hline \multicolumn{6}{|l|}{$m_{e \mu}<2.5 \mathrm{eV}$} \\
\hline$\lambda_{133}^{\prime} \lambda_{233}^{\prime}$ & $3.8 \cdot 10^{-7}$ & $3.5 \cdot 10^{-5} 19$ & 0 & 0 & 0 \\
\hline$\lambda_{132}^{\prime} \lambda_{223}^{\prime}$ & $9.3 \cdot 10^{-6}$ & $2.4 \cdot 10^{-2}$ & $1.1 \cdot 10^{-15}$ & $7.2 \cdot 10^{-16}$ & $1.8 \cdot 10^{-15}$ \\
\hline$\lambda_{123}^{\prime} \lambda_{232}^{\prime}$ & $9.3 \cdot 10^{-6}$ & $1.5 \cdot 10^{-2}$ & $1.1 \cdot 10^{-15}$ & $1.8 \cdot 10^{-15}$ & $7.2 \cdot 10^{-16}$ \\
\hline$\lambda_{122}^{\prime} \lambda_{222}^{\prime}$ & $2.3 \cdot 10^{-4}$ & $3.3 \cdot 10^{-7} 19$ & 0 & 0 & 0 \\
\hline$\lambda_{133} \lambda_{233}$ & $6.6 \cdot 10^{-6}$ & $1.7 \cdot 10^{-5} 19$ & 0 & 0 & 0 \\
\hline$\lambda_{123} \lambda_{232}$ & $1.1 \cdot 10^{-4}$ & $2.0 \cdot 10^{-3}$ & $2.9 \cdot 10^{-15}$ & $6.0 \cdot 10^{-15}$ & $3.1 \cdot 10^{-15}$ \\
\hline \multicolumn{6}{|l|}{$m_{\mu \mu}<2.5 \mathrm{eV}$} \\
\hline$\lambda_{233}^{\prime} \lambda_{233}^{\prime}$ & $3.8 \cdot 10^{-7}$ & $1.4 \cdot 10^{-1}$ & 0 & 0 & 0 \\
\hline$\lambda_{232}^{\prime} \lambda_{223}^{\prime}$ & $9.3 \cdot 10^{-6}$ & $2.2 \cdot 10^{-2}$ & 0 & 0 & 0 \\
\hline$\lambda_{222}^{\prime} \lambda_{222}^{\prime}$ & $2.3 \cdot 10^{-4}$ & $3.6 \cdot 10^{-3}$ & 0 & 0 & 0 \\
\hline$\lambda_{233} \lambda_{233}$ & $6.6 \cdot 10^{-6}$ & $2.5 \cdot 10^{-3}$ & 0 & 0 & 0 \\
\hline \multicolumn{6}{|l|}{$m_{e \tau}<2.5 \mathrm{eV}$} \\
\hline$\lambda_{133}^{\prime} \lambda_{333}^{\prime}$ & $3.8 \cdot 10^{-7}$ & $1.2 \cdot 10^{-4}$ & 0 & 0 & 0 \\
\hline$\lambda_{132}^{\prime} \lambda_{323}^{\prime}$ & $9.3 \cdot 10^{-6}$ & $1.4 \cdot 10^{-1}$ & $1.1 \cdot 10^{-15}$ & $7.2 \cdot 10^{-16}$ & $1.8 \cdot 10^{-15}$ \\
\hline$\lambda_{123}^{\prime} \lambda_{332}^{\prime}$ & $9.3 \cdot 10^{-6}$ & $6.8 \cdot 10^{-3}$ & $1.1 \cdot 10^{-15}$ & $1.8 \cdot 10^{-15}$ & $7.2 \cdot 10^{-16}$ \\
\hline$\lambda_{122}^{\prime} \lambda_{322}^{\prime}$ & $2.3 \cdot 10^{-4}$ & $7.2 \cdot 10^{-3}$ & 0 & 0 & 0 \\
\hline$\lambda_{132} \lambda_{323}$ & $1.1 \cdot 10^{-4}$ & $2.5 \cdot 10^{-3}$ & $2.9 \cdot 10^{-15}$ & $3.1 \cdot 10^{-15}$ & $6.0 \cdot 10^{-15}$ \\
\hline$\lambda_{122} \lambda_{322}$ & $1.8 \cdot 10^{-3}$ & $2.0 \cdot 10^{-3}$ & 0 & 0 & 0 \\
\hline \multicolumn{6}{|l|}{$m_{\mu \tau}<2.5 \mathrm{eV}$} \\
\hline$\lambda_{233}^{\prime} \lambda_{333}^{\prime}$ & $3.8 \cdot 10^{-7}$ & $6.3 \cdot 10^{-2}$ & 0 & 0 & 0 \\
\hline$\lambda_{232}^{\prime} \lambda_{323}^{\prime}$ & $9.3 \cdot 10^{-6}$ & $1.3 \cdot 10^{-1}$ & $1.1 \cdot 10^{-15}$ & $7.2 \cdot 10^{-16}$ & $1.8 \cdot 10^{-15}$ \\
\hline$\lambda_{223}^{\prime} \lambda_{332}^{\prime}$ & $9.3 \cdot 10^{-6}$ & $1.0 \cdot 10^{-2}$ & $1.1 \cdot 10^{-15}$ & $1.8 \cdot 10^{-15}$ & $7.2 \cdot 10^{-16}$ \\
\hline$\lambda_{222}^{\prime} \lambda_{322}^{\prime}$ & $2.3 \cdot 10^{-4}$ & $2.2 \cdot 10^{-2}$ & 0 & 0 & 0 \\
\hline$\lambda_{232} \lambda_{323}$ & $1.1 \cdot 10^{-4}$ & $2.5 \cdot 10^{-3}$ & $2.9 \cdot 10^{-15}$ & $3.1 \cdot 10^{-15}$ & $6.0 \cdot 10^{-15}$ \\
\hline \multicolumn{6}{|l|}{$m_{\tau \tau}<2.5 \mathrm{eV}$} \\
\hline$\lambda_{333}^{\prime} \lambda_{333}^{\prime}$ & $3.8 \cdot 10^{-7}$ & $2.9 \cdot 10^{-2}$ & 0 & 0 & 0 \\
\hline$\lambda_{332}^{\prime} \lambda_{323}^{\prime}$ & $9.3 \cdot 10^{-6}$ & $6.1 \cdot 10^{-2}$ & 0 & 0 & 0 \\
\hline$\lambda_{322}^{\prime} \lambda_{322}^{\prime}$ & $2.3 \cdot 10^{-4}$ & $1.3 \cdot 10^{-1}$ & 0 & 0 & 0 \\
\hline$\lambda_{322} \lambda_{322}$ & $1.8 \cdot 10^{-3}$ & $2.5 \cdot 10^{-3}$ & 0 & 0 & 0 \\
\hline
\end{tabular}


Figure 1: The $\lambda^{\prime}$-induced one loop diagrams contributing to Majorana masses for the neutrinos. To generate magnetic moments photons should be attached to the internal lines.

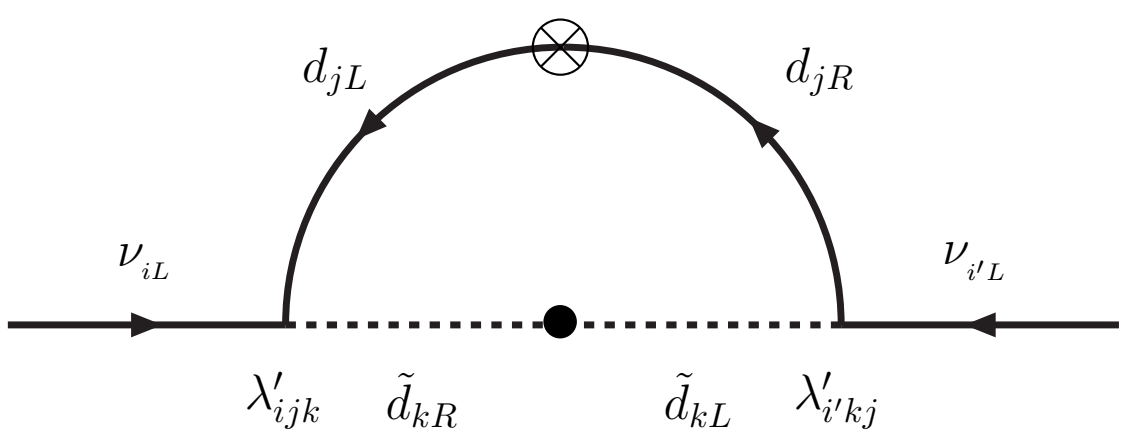

(a)

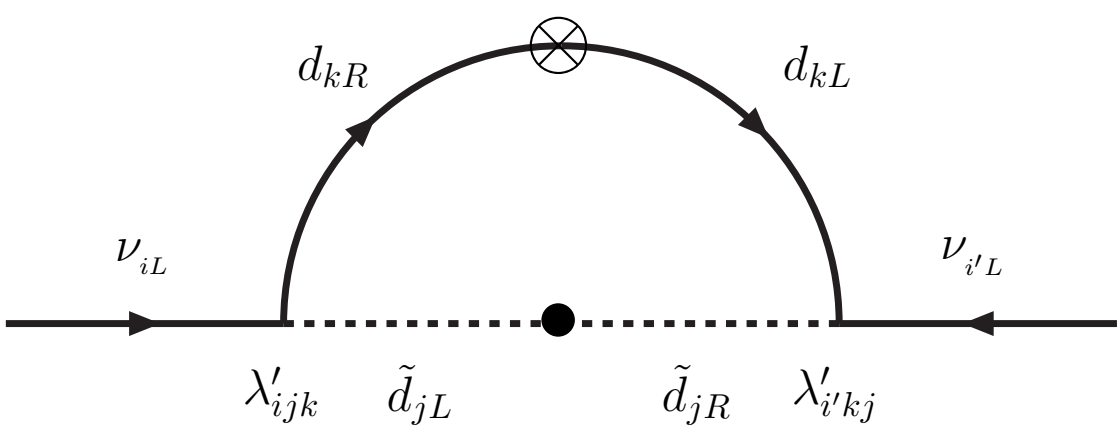

(b) 